Experimental results on quantum chromo dynamics: what is next?

This content has been downloaded from IOPscience. Please scroll down to see the full text. 2013 Phys. Scr. 2013014001

(http://iopscience.iop.org/1402-4896/2013/T158/014001)

View the table of contents for this issue, or go to the journal homepage for more

Download details:

IP Address: 137.138.125.164

This content was downloaded on 21/01/2014 at 11:42

Please note that terms and conditions apply. 


\title{
Experimental results on quantum chromo dynamics: what is next?
}

\author{
Albert De Roeck \\ CERN, CH-1211 Geneva 23, Switzerland \\ and \\ University of Antwerp, Groenenborgerlaan 171, B-2020 Antwerp, Belgium \\ E-mail: Albert.de.roeck@cern.ch
}

Received 1 October 2013

Accepted for publication 6 November 2013

Published 6 December 2013

Online at stacks.iop.org/PhysScr/T158/014001

\begin{abstract}
This review gives a flavour of experimental quantum chromo dynamics (QCD) results obtained at the Large Hadron Collider (LHC) during the first run period in the years 2010-2012. The results cover selected aspects of soft low- $p_{\mathrm{T}}$ phenomena, typically described by phenomenological models, as well as high- $p_{\mathrm{T}}$ processes which can be studied theoretically with perturbative techniques. In general the phenomenological models required tuning to describe the data in the new energy region of 7-8 TeV, while perturbative QCD (pQCD) is found to work generally quite well for most of the phase space currently studied. The strong force will remain a main topic of research at colliders such as the LHC also in future, with the large data samples allowing for more detailed studies and in particular when the next energy level of $13-14 \mathrm{TeV}$ will be reached.
\end{abstract}

PACS numbers: 12.38.-t, 13.85.- t, 13.87.-a

(Some figures may appear in colour only in the online journal)

\section{Introduction}

Quantum chromo dynamics (QCD), the strong force acting between quarks and gluons, plays a major role in basically every topic discussed at this Symposium on the Large Hadron Collider (LHC) physics results. For precision measurements and for searches for new physics we need to understand the role and effects of QCD. After all, QCD is all around us at the LHC [1]!

In this review we will touch upon the following subjects: soft QCD dynamics measurements such as particle production and fragmentation; correlations between particles and possible new effects; forward energy measurements; and elastic, diffractive and total cross sections measurements. QCD has been mostly studied in the perturbative regime at the LHC. Results are discussed on jet production, jet substructure and the extraction of $\alpha_{\mathrm{s}}$. Other measurements include the structure of the proton and low- $x$ studies.

In this report only published data of the LHC experiments are presented. It should be noted that six out of the seven experiments at the LHC have published results on QCD-related topics. Many of the techniques discussed here have been pioneered at previous colliders, in particular the
Tevatron, but the higher energy and large event rate of the LHC elevates the QCD studies to a new height, with several novel analysis avenues that have been opened in the last two years.

\section{Soft QCD dynamics}

Most $p p$ collisions at the LHC are soft, i.e. these do not involve a hard scale. Typical transverse momenta, $p_{\mathrm{T}}$, of the particles emerging from the interactions are of the order of a few $100 \mathrm{MeV}$. These processes constitute the largest part of the $p p$ cross section and hence of the so called pile-up events in the experiments. These are events that are produced simultaneously in a bunch crossing together with the main triggered hard scattering interaction of interest. The pile-up has increased with time at the LHC for the experiments ATLAS and CMS, reaching about 20 events per bunch-crossing on average at the highest luminosities in 2012. In future we expect the pile-up to increase even further as the instantaneous luminosity will increase.

Pile-up is treated in the experiments by overlaying minimum bias Monte Carlo events, i.e. events dominated 


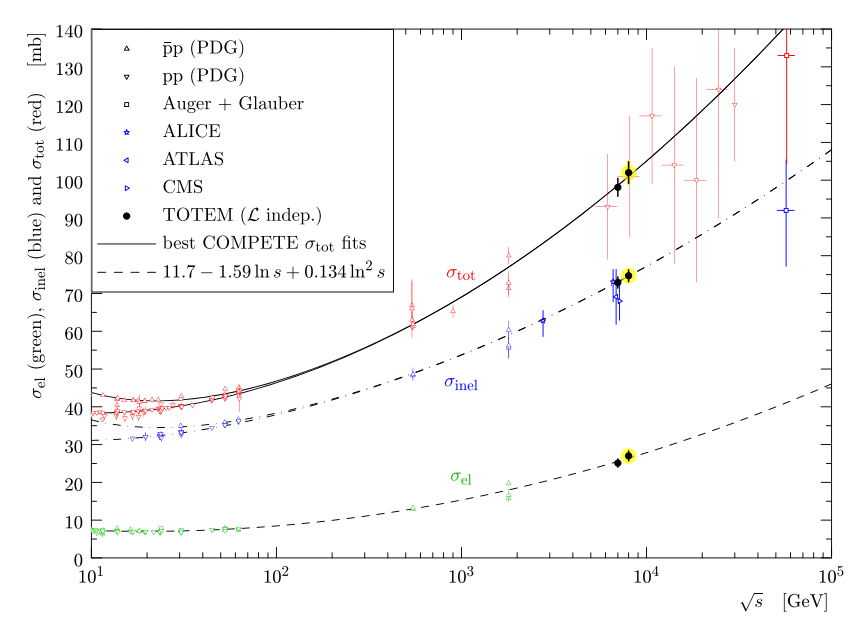

Figure 1. Compilation of the total, inelastic and elastic cross section measurements. The black lines represent the best fits produced by the COMPETE collaboration.

by soft QCD interactions using phenomenological models such as PYTHIA [2] and HERWIG [3], on top of the signal samples. These generators are typically tuned to data taken during low pile-up conditions of the LHC-typically at the very beginning of the run- and the multi-particle production characteristics are matched to measured the ones in those data.

Proton-proton collisions can be divided into at least four categories, namely elastic scattering, single diffractive dissociation, double diffractive dissociation and inelastic non-diffractive collisions. It is not always possible to clearly identify experimentally to which category an event belongs; typically rapidity gaps and forward proton tagging techniques are used to help to classify events.

Most LHC experiments are insensitive to the elastic scattering part of the cross sections because the scattered protons remain in the beam-pipes. TOTEM [4] is a dedicated experiment for the measurement of total, elastic and soft diffractive cross sections. This experiment consists of near beam detectors and forward detectors with an acceptance $|\eta|>3$, where $\eta$ is defined as $\eta=-\ln \tan \theta / 2$ with $\theta$ the polar angle of a particle measured in the laboratory frame. The near beam detectors, in this case Roman Pots, are the relevant ones for the measurement of the elastic scattering cross section. TOTEM has measured elastic cross sections as $\mathrm{d} \sigma / \mathrm{d}|t|$ distributions [5], with $t$ the momentum transfer squared of the elastic scattering.

An important measurement at any new energy is the total $p p$ cross section. TOTEM has used several different techniques to extract the total cross section, and the one using the optical theorem, relating the forward scattering amplitude to the total cross section, leads to $\sigma_{\text {tot }}=101.7 \pm$ $2.9 \mathrm{mb}$. Furthermore the elastic and inelastic parts of the total cross section are measured to be $27.1 \pm 1.4$ and $74.7 \pm 1.7 \mathrm{mb}$ respectively [6]. The results are shown in figure 1 . There is a smooth continuous rise of the total cross section with centre of mass energy, and e.g. no sudden strong rise in the LHC region, as was predicted in some more exotic models. These measurements will certainly be repeated at 13 (14) TeV and also further improvements in LHC operation may allow for high $\beta$ optics measurements that will allow to probe the Coulomb-Nuclear interference region.
Other LHC experiments have made estimates of the total cross section as well. These measurements typically make use of extrapolations of a cross section measured in a visible region $[7,8]$ to a full phase space cross section, and therefore have considerable uncertainties, but are found to be in general in good agreement with the TOTEM measurement. Experiments have also provided the first, albeit not yet very precise measurements on the single and double diffractive components in the data, see e.g. the ALICE results in [9]. The result are as follows. The fractions of diffractive processes in inelastic collisions were determined from a study of gaps in charged particle pseudorapidity distributions: for single diffraction (diffractive mass $M_{X}<200 \mathrm{GeV} / c^{-2}$ ) $\sigma_{\mathrm{SD}} / \sigma_{\mathrm{INEL}}=0.21 \pm 0.03,0.20_{-0.08}^{+0.07} \quad$ and $\quad 0.20_{-0.07}^{+0.04}$, respectively at centre-of-mass energies $\sqrt{s}=0.9,2.76$ and $7 \mathrm{TeV}$; for double diffraction (for a pseudorapidity gap $\Delta \eta>3) \sigma_{\mathrm{DD}} / \sigma_{\mathrm{INEL}}=0.11 \pm 0.03,0.12 \pm 0.05$ and $0.12_{-0.04}^{+0.05}$, respectively at $\sqrt{s}=0.9,2.76$, and $7 \mathrm{TeV}$. Diffractive studies at the LHC are only at the beginning and there are yet a lot of results to be expected in this field in the coming years.

In case of the occurrence of a hard scattering in the event we also identify a so called underlying event on top of the hard process, part of which comes from the proton remnants not taking part in the hard scattering leading to possible additional, by definition softer, scatterings among these remnants. The precise understanding of the dynamics of the underlying event is essential for some precision measurements. An example is the top mass determination. QCD is now responsible for most of the uncertainty on the measurement: non-perturbative QCD effects including hadronization, colour reconnection and in particular the underlying event tunes are the dominant uncertainties.

All four central LHC experiments have produced measurements of the underlying event activity in collisions which have a (semi-)hard scattering. Typically measurements are made in the transverse region away from the hard scattering activity, and a growth of this away activity is studied as function of the hard scale in the scattering. It is found that the underlying event activity is more than a factor two larger for $7 \mathrm{TeV}$ collisions compared to collisions at $900 \mathrm{GeV}$, as determined with the help of a data sample collected before the LHC ramped up to high energy. These measurements have allowed for new Monte Carlo tunes to be produced, which describe many aspects of the data, and are now used as a standard tune for the simulations for underlying event activities. While starting with tracks and jets, these analyses have now evolved to more detailed studies and e.g. combine jets with photon final states [11].

An important component for the study of soft QCD dynamics is the detailed understanding of particle production. CMS, ATLAS, ALICE and LHCb have produced a plethora of results on charged multiplicity distributions, single particle measurements, strange particle production, baryon and anti-baryon production and more. In many ways the different types of LHC detectors are excellent for such measurements, and very complimentary in their capabilities. Discrepancies have been observed between data and the fragmentation in phenomenological models predictions. As an example figure 2 (left) shows the forward $\phi$ meson production rate measured in $\mathrm{LHCb}$ [10]. A tune that describes all aspects of particle 

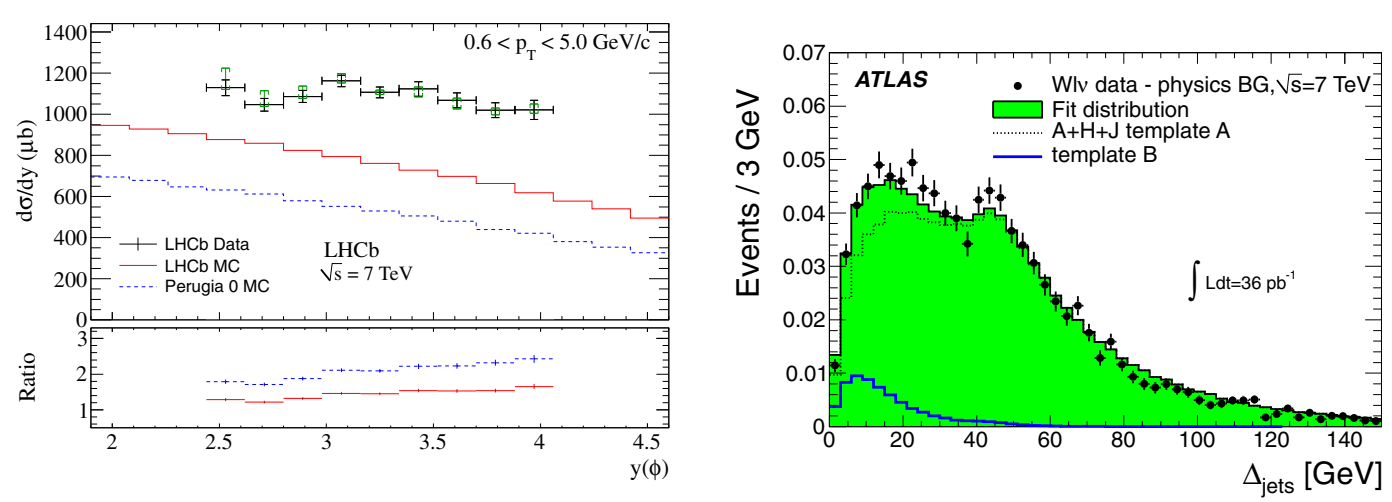

Figure 2. (left) Inclusive differential $\phi$ production cross section as a function of rapidity, measured with data (points), and compared to the $\mathrm{LHCb}$ default Monte Carlo (MC) tuning (solid line) and Perugia-0 tuning (dashed line). The error bars represent the statistical uncertainty, the braces show the bin dependent systematic errors. The lower part of the plot shows the ratio data cross section over Monte Carlo cross section. (right) Distribution of $\Delta_{\text {Iets }}$ in the background-subtracted data (dots) compared to the result from the best fit. The result is shown as the green histogram. Data and the overall fit have been normalized to unity.

production at 7 or $8 \mathrm{TeV}$ is not available yet. Hence a systematic approach is needed to organize one-or perhaps a few-more universal tunes of Monte Carlos programmes. This will also help to set up the tools for getting a new tune fast when the machine comes back in 2015 at higher energy. Such a tune will be imperative for e.g. estimating accurately the effects of pile-up, which is expected to become important very soon for run-II.

Of particular interest for Monte Carlo tunes, but even more so for searches for rare event topologies, is the phenomenon of double parton scattering (DPS) within one and the same collision. In DPS events there are (at least) two hard or semi-hard scatterings from different partons in the two incoming protons. An example is the recent ATLAS analysis [12] that studies the angular correlations in $W$ plus dijet events, in order to kinematically separate the basic process ' $W+$ dijets' from the DPS processes: one collision producing a $W$ and the other producing a dijet. This analysis showed that the contribution of the DPS processes in the kinematic range considered could be quite substantial: the measured fraction is $0.08 \pm 0.01$ (stat.) \pm 0.02 (sys.) of the total $W$ plus dijet rate production. The data are shown in figure 2 (right). Hence DPS can be important for searches for new phenomena, where after cuts only a few events are expected to remain. What possible additional measurements can we make at the LHC to improve our understanding and control DPS?

Measurements of correlations between particles have traditionally been a rich source of information for the understanding of multi-particle dynamics. In the early days of the first LHC data the experiments have already studied correlations. An interesting and unexpected effect was found by CMS when studying two particle angular correlations [13]. The difference in the angles $\Delta \phi$ (transverse) and $\Delta \eta$ (longitudinal) between each pair of charged particles is measured and resulting correlations determined. Such correlations have been studied in $p p$ collisions before and one expects a positive correlation for the region $\Delta \phi \sim 0$ and $\Delta \eta \sim 0$ due to short range effects and jet formation, plus a positive correlation for all $\Delta \eta$ at $\Delta \phi \sim \pi$, which reflects the effect of the balancing jet in two jet events. Much to the surprise, a weak but definite positive correlation was also found for
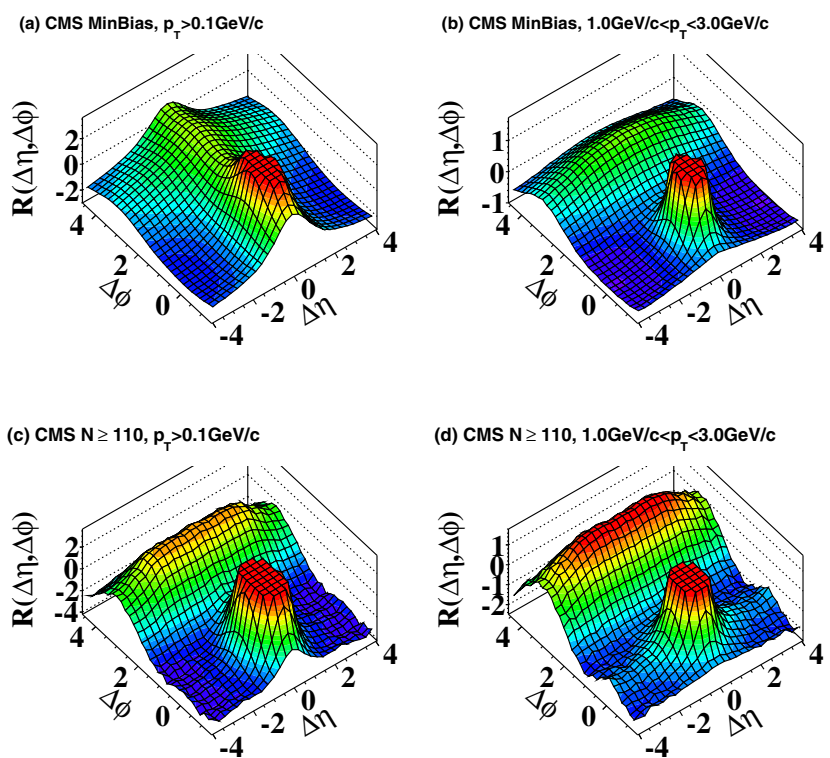

Figure 3. Two-dimensional two-particle correlation functions for $7 \mathrm{TeV} p p$ (a) minimum bias events with $p_{\mathrm{T}}>0.1 \mathrm{GeV}$,

(b) minimum bias events with $1<p_{\mathrm{T}}<3 \mathrm{GeV}$, (c) high multiplicity $\left(N_{\text {trk }}^{\text {offine }} \geqslant 110\right)$ events with $p_{\mathrm{T}}>0.1 \mathrm{GeV}$ and (d) high multiplicity $\left(N_{\text {trk }}^{\text {offline }} \geqslant 110\right)$ events with $1<p_{\mathrm{T}}<3 \mathrm{GeV}$. The sharp near-side peak from jet correlations is cut off in order to better illustrate the structure outside that region.

all $\Delta \eta$ for $\Delta \phi \sim 0$, but only in events with a high charged particle multiplicity, namely close to a 100 charged particles! This effect is shown in figure 3. This behaviour was not predicted by the phenomenological models used for LHC QCD physics, and its origin is still a bit enigmatic. It suggests the presence of a new phenomenon of the strong force. Models based on colour glass condensates or hydrodynamic models may offer an explanation, but also a -perhaps unnaturally-large amount of multiple interactions seems to work [14]. Do we need new additional measurements to understand this possible new QCD phenomena further, and if so, which ones?

Finally, let us turn now to a few specific forward measurements. TOTEM was mentioned before; it uses the same interaction point as the CMS experiment (IP5) and is mechanically integrated with this experiment. Its forward 

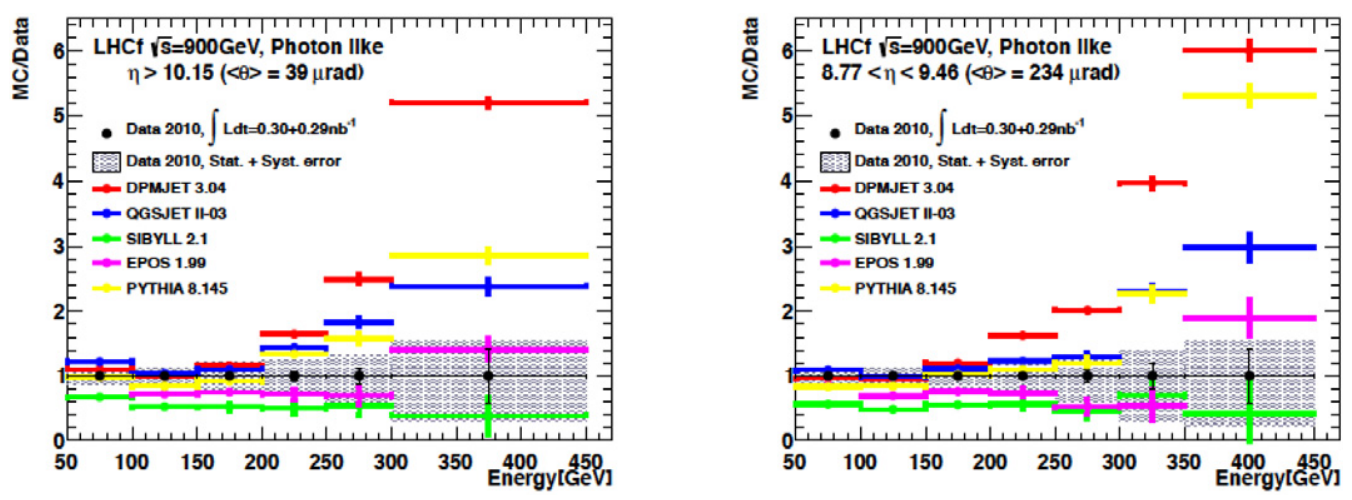

Figure 4. Ratio of the MC spectra divided by the data in each energy bin. The left and the right panels show the spectra for the pseudorapidity ranges of $\eta>10.15$ and $8.77<\eta<9.46$, respectively. The colored plots indicate the results of Monte Carlos used in cosmic ray studies.

telescopes with acceptance of roughly $3.0 \leqslant|\eta| \leqslant 6.5$ detect charged particles where CMS does not have tracking coverage. CMS has however calorimetric coverage till $|\eta|=$ 5.0 and on one side even till $|\eta| \sim 7.0$ owing to the CASTOR calorimeter. Hence TOTEM and CMS together have a large complimentary forward coverage. In addition TOTEM has Roman Pot detectors at about 147 and $220 \mathrm{~m}$ from the IP. Hence it is useful to organize common data runs between the two experiments. This was for the first time accomplished in 2011, for a few low luminosity runs. In 2012 a total of $10 \mathrm{nb}^{-1}$ has been collected in addition. Offline the commonly triggered data could be identified in the two separate experimental data streams and combined for analyses. There are many physics opportunities for such a combined central and forward large acceptance detector, such as the study of events with a hard scatter, e.g. with jets, in the central detectors and with tagged protons.

One of the interesting processes postulated a few years ago was to detect exclusively produced Higgses [15, 16], i.e. the process $p p \rightarrow p+H+p$. If measurable, this process can play a significant role in the Higgs physics programme. The scattered protons need to be detected and measured in near beam detectors. Since this process involves only three particles in the final state, one can determine the signal of the $H$ from the missing mass to the beam proton momenta, independent of the decay of the Higgs itself. The achievable mass resolution calculated from the scattered protons is $1-2 \%$. Given that we know now that the mass is around $126 \mathrm{GeV}$, one can select a mass interval for the Higgs events. Moreover in such an exclusive process the QCD $b b$ background is strongly suppressed, allowing for a direct observation of the Higgs to $b b$ decay channel. Also the azimuthal proton-proton correlations can be used as a powerful $\mathrm{CP}$ analyser of the Higgs quantum numbers. There are at least two drawbacks right now for these measurements. Firstly for Standard Model Higgs production processes the cross section is only $5-10 \mathrm{fb}^{-1}$, i.e. when all efficiencies are taken into account, a few 10's of events for a luminosity of $100 \mathrm{fb}^{-1}$ will be collected. MSSM Higgs production scenarios can give like a ten times higher rate, but the non-observation of supersymmetry (SUSY) so far at the LHC does start to close these opportunistic windows [17]. Secondly, the experiments need near beam detectors at about $420 \mathrm{~m}$ away from the IP, in the cold section of the machine. While probably technically feasible as shown in an initial study [18] it will require a lot of effort to realize this, and presently it is not yet clearly on a definite roadmap of the experiments. Hence it is at this point clear that, if ever, such measurements will be realized only in some further future.

Another experiment that is entirely tailored for forward measurement is LHCf. This experiment uses the same interaction point as ATLAS (IP1). LHCf has forward electromagnetic calorimeters positioned literally at zero degrees, as seen from the IP, and located at $140 \mathrm{~m}$. The physics aim is to measure very forward photons and neutral pions, and these measurement results are primarily targeted for improving cosmic ray studies, by contributing to a better understanding and tuning of the cosmic ray shower Monte Carlos. These are used to determine for a cosmic shower, based on ground based measurements, what the original energy and type of incident particle of the cosmic ray was when it hit the earth atmosphere. LHCf has produced results for the $900 \mathrm{GeV}$ and $7 \mathrm{TeV} p p$ run and an example is given in figure 4 [19]. Interestingly enough, none of the current Monte Carlo models used in the cosmic ray community reproduces the data well! Clearly new tunes can be provided with these LHCf data but it generally illustrates the importance of making forward measurements at the LHC, so these can be used to improve the Monte Carlo modelling. Recently a working group was initiated to explore further opportunities of LHC measurements from all experiments that can be useful in this context.

\section{Hard scattering and perturbative QCD}

Perturbative QCD can be applied to hard scattering processes such as jet production. Jets of particles, collimated around the original parton direction, emerge after a hard energy parton-parton scattering. The ATLAS and CMS experiments have now observed events with dijet invariant masses larger than $5 \mathrm{TeV}$. This means that for such events more than $60 \%$ of the full proton-proton energy ends up in jets, which is remarkable if one remembers that the energy of the proton is shared by several partons in the proton.

Jets were observed in the early days of the LHC running, already with relatively low accumulated integrated luminosity. For example ATLAS published a study on multi-jet events in 

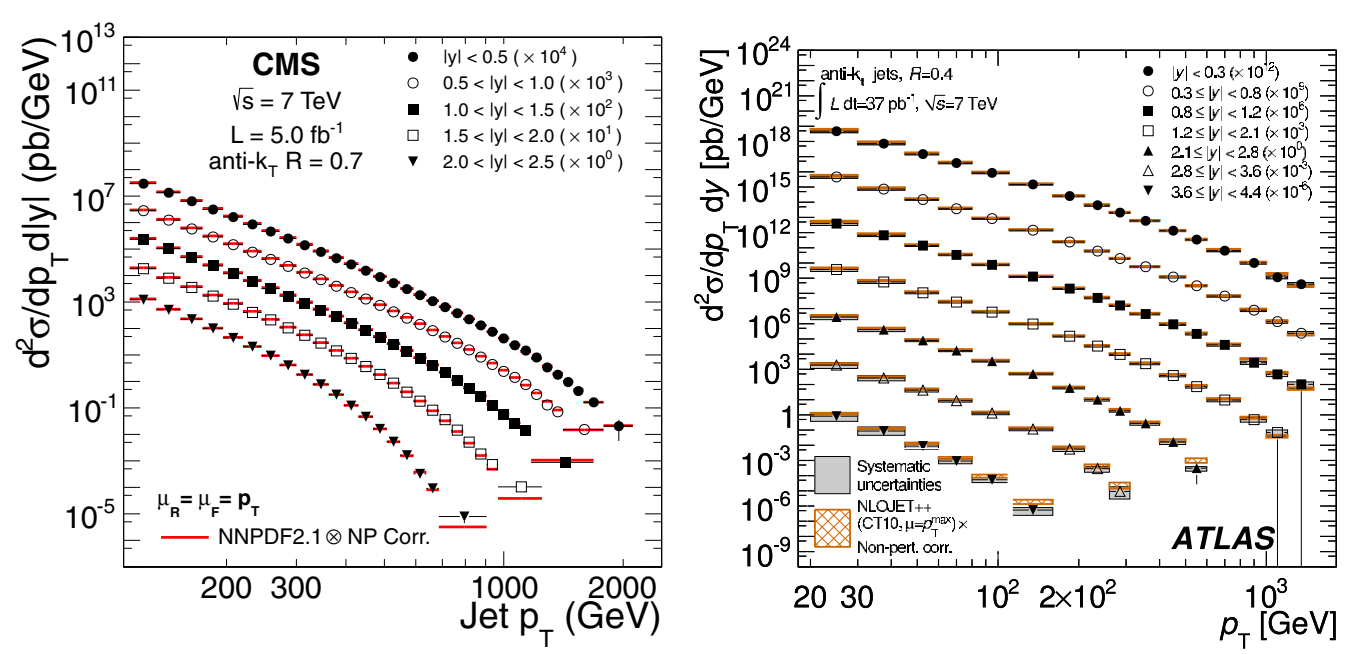

Figure 5. (Left) Inclusive jet cross sections for five different rapidity bins by CMS, using the NNPDF2.1 PDF set. The anti-kT algorithm with $R=0.5$ was used. (Right) Inclusive jet cross sections for seven different rapidity bins by ATLAS, compared to NLO pQCD predictions from NLOJET++ plus non-perturbative corrections. The anti- $k T$ algorithm with $R=0.4$ was used. The theory uncertainty, shown as the light, hatched band, is the quadratic sum of uncertainties from the choice of the renormalization and factorization scales, PDFs, $\alpha_{\mathrm{s}}(M Z)$, and the modelling of non-perturbative effects.

2010 with just $2.4 \mathrm{pb}^{-1}$ of data [20]. Jet multiplicities of up to six jets were reported and the relative rates shown to be in good agreement with theory, which consisted of leading order matrix elements plus matched parton showers. Inclusive jet spectra have been shown by CMS [21] and ATLAS [22] as function of the jet $p_{\mathrm{T}}$ for different regions in rapidity. An example of the data for $7 \mathrm{TeV}$ is shown in figure 5. Agreement with next to leading order (NLO) calculations is observed over the full range, up to $2 \mathrm{TeV}$ jets. Dijet mass distributions show an equal good agreement with the LHC data. The anti-kT jet algorithm is used in most studies by both experiments. The cone chosen for this algorithm is however different for ATLAS and CMS so these measurements are not directly comparable. ATLAS uses a distance parameter of $R=0.4$ or 0.6 for jet studies while CMS uses $R=0.5$ or 0.7 . Making the two experiments use the same parameter in future seems a difficult challenge!

There is some evidence that the agreement with the data seems to become worse, albeit within large uncertainties, for the highest probed rapidity range, something to watch and study in more detail in future. The precision is limited by the systematic uncertainties on the measurements, in particular by the experimental energy scale uncertainties. This will also hamper using the jet results to improve on the parton distributions, as discussed below. A way to reduce the systematics is by studying ratios of jet cross sections at different centre of mass energies. ATLAS compares data from proton-proton collisions at 2.76 and $7 \mathrm{TeV}$ in [23], and many systematic uncertainties cancel partially or completely. More measurements of this type may become important in future.

Azimuthal correlations between the two most leading jets are sensitive to the QCD radiation in the event, both initial and final state radiation, as demonstrated in the ATLAS [24] and CMS [25] analyses. Initial state radiation (ISR) has become very important for searches for new physics at the LHC, and a good understanding of this effect is imperative for some key analyses. Examples are the dark matter searches and searches for SUSY particles. Analyses of mono-jets and mono-photons can be used to search dark matter. The basic diagram is the production of a pair of dark matter particles from quark-anti-quark or gluon fusion. This final state will not give any measurable signature in the detector, but if there is an initial state gluon or photon emitted, the topology of the event will look like a single jet or photon with large missing balancing transverse energy. Using effective contact theories one can derive limits on spin-dependent and -independent cross sections of dark matter particles with baryonic matter, which compare well with those of direct searches as shown in figure 6, see e.g. [26]. Note however that recently there has been some criticism on the region of validity of this comparison of collider results with direct searches [27]. In any case, such studies rely critically on the understanding of the ISR.

Similarly some SUSY searches with compressed spectra rely on ISR, where the events are actually triggered by the ISR in the event. An example is the AMSB study with semi-stable gauginos as presented in [28]. This underlines further the need for a good understanding of ISR at the LHC, and new ideas for experimental tests of ISR will be of help.

As a spin-off of QCD measurements tests can be made on the elementariness of the quarks. The measurement of the production angle of the jets with respect to the beam-axis is sensitive to possible substructure in the quarks, in a similar way the Rutherford experiment discovered the existence of nuclei in atoms. Angular analyses with jets have been performed and so far no hint of a substructure of quarks has been identified. The limits are now for compositeness scales to be larger than $7.5 \mathrm{TeV}$. Thus, quarks remain elementary particles after these measurements at the highest energies in the laboratory.

A new era of QCD measurements has opened at the LHC with precision measurements of vector bosons and top quarks with associated jets. For examples $Z$ boson with up to seven associated jets have been measured [29] and top samples with in total eight jets [30] have been identified; both measurements are described well by theory. The processes are 

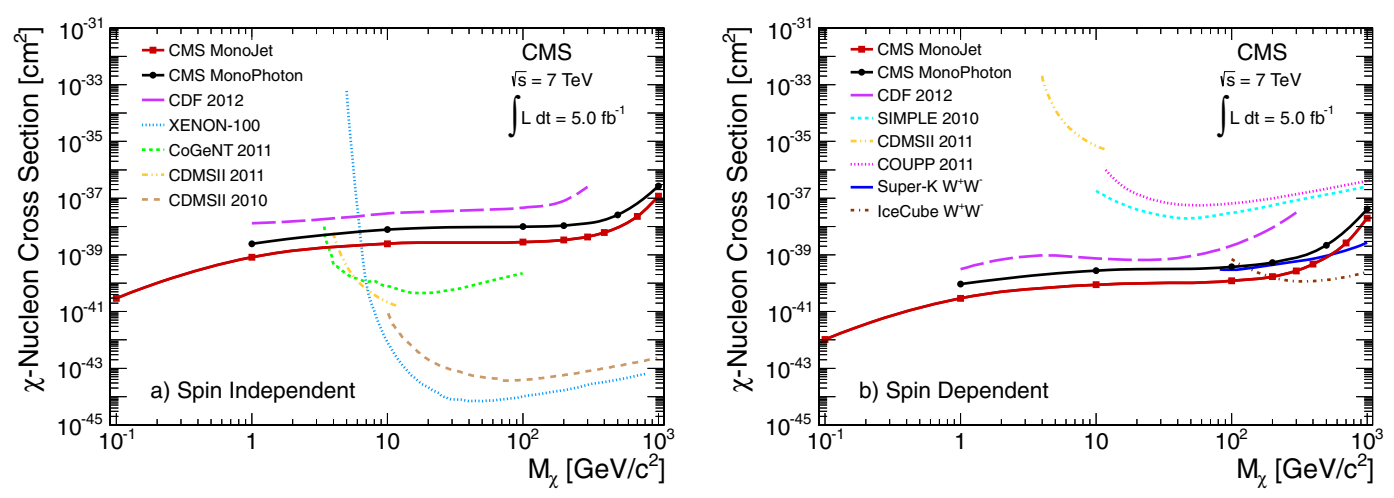

Figure 6. Comparison of the $90 \%$ CL upper limits on the dark matter-nucleon scattering cross section versus dark matter mass for the spin-independent and spin-dependent models with results from various direct detection experiments.
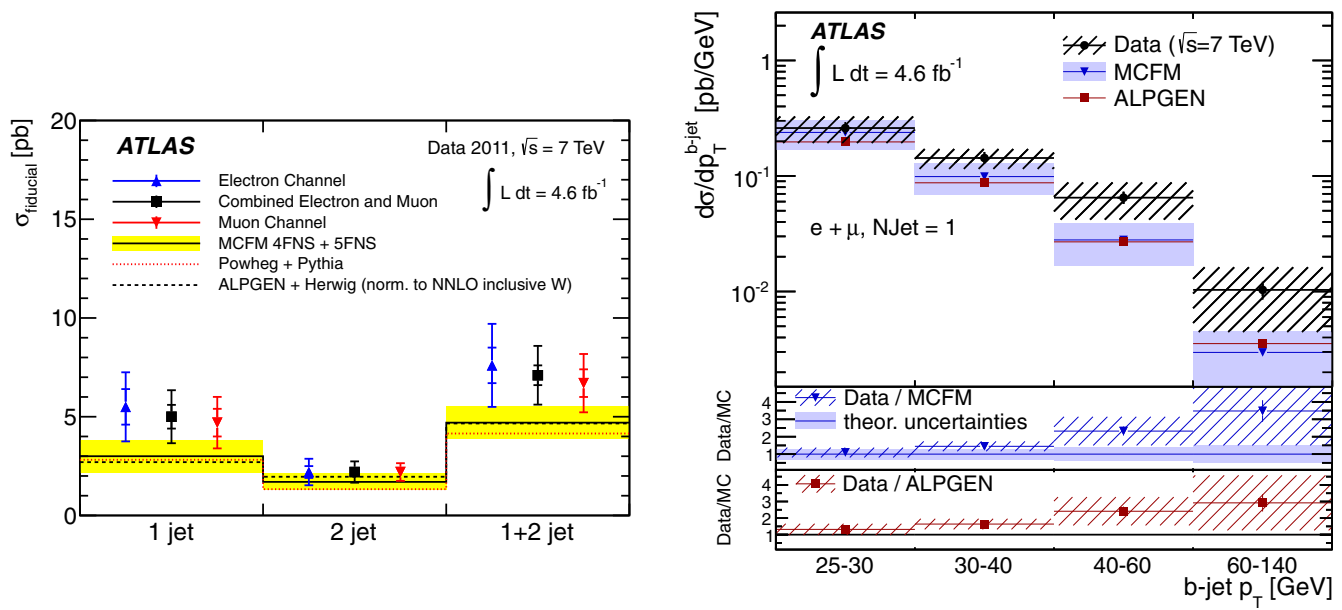

Figure 7. (Left) Measured fiducial cross sections with the statistical (inner error bar) and statistical plus systematic (outer error bar) uncertainties in the electron, muon and combined electron and muon channels. The cross sections are given in the one-jet, two-jet and one + two-jet fiducial regions. The measurements are compared with NLO predictions calculated with MCFM and corrected for hadronization and DPS effects. The yellow bands represent the total uncertainty on the prediction. (Right) Measured differential $W+b$-jets cross sections with the statistical plus systematic uncertainties as a function of $p_{\mathrm{T}}(b)$ in the one-jet and two-jet fiducial regions, obtained by combining the muon and electron channel results. The measurements are compared to the MCFM predictions and to the ALPGEN predictions interfaced to HERWIG and JIMMY and scaled by the NNLO inclusive $W$ normalization factor.

important backgrounds for searches for new physics, e.g. for SUSY searches, so a good theoretical understanding of these backgrounds generates confidence in the analyses techniques used for these searches. Note that so far mostly $7 \mathrm{TeV}$ data based result have been released by the experiments on many of these measurements, hence there is still a large potential for new more precise results to come soon. Similarly data on hard prompt photon production, not discussed here, also play an important role in tests for QCD.

The LHC experiments have excellent $b$-tagging capabilities, which allows for further sophisticated measurements such as vector boson production with associated $b$-jets. Data in both experiments shows that the $W+1 b$-jet cross section is rather on the high side, while the $W+2 b$-jets cross sections agree better with predictions. As an example we shown in figure 7 the ATLAS result [31]. Future measurements will referee whether this tendency will persist and will need a more detailed investigation.

The proton is a composite particle consisting of quarks and gluons. Parton distribution functions (PDFs) are therefore an essential ingredient of the LHC physics programme. Several PDF families are now available at NNLO precision and are used by the experiments to confront with data: ABM [32], MSTW [34], CT [33], NNPDF [35], HERAPDF [36]. Measurements sensitive to PDFs themselves are studied within the experiments, and across experiments with the PDF-fitting groups in the PDF4LHC study group [37]. The PDF-fitting groups provide both central values and uncertainty envelopes for the parton distributions, and they differ in detail as a result of the different choice of data used as input for the fits, different theoretical treatment for certain aspects e.g. for heavy flavours, etc. The PDF precision impacts LHC physics: e.g. for most of the processes for Higgs production the PDFs are an important contribution to the uncertainties, as shown in figure 8 (left) [38]. For example for gluon-gluon fusion $50 \%$ of the error (which is in total 15\%) stems from the PDFs and the strong coupling constant. So it is imperative that we improve on the precision of the PDFs in future. Final precise HERA data may help here, but also the LHC data itself will allow to improve the PDFs further. In particular vector boson production and $W$-asymmetry data, top quark data, data of $W+$ charm quarks, prompt photon data and perhaps the di-jet data-if the systematic uncertainties can be reduced-will play an 

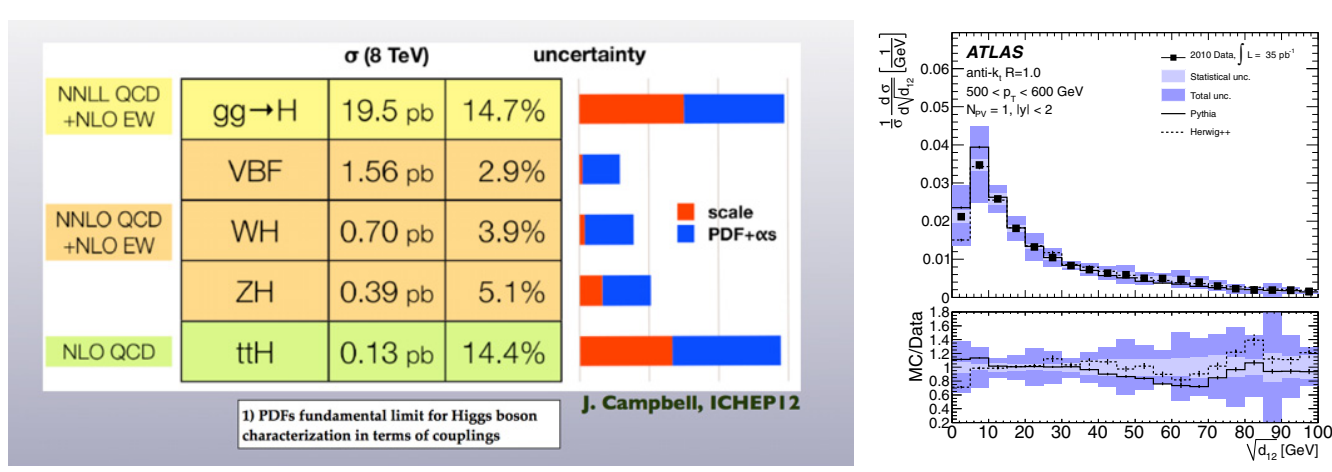

Figure 8. (Left) Uncertainties on the different Higgs production processes, and a visual presentation on the fraction of the PDF and scale contributions to the total uncertainty. (Right) Normalized cross section as a function of scale splitting variable $\sqrt{d_{12}}$ of anti- $k T$ jets with $R=1.0$.
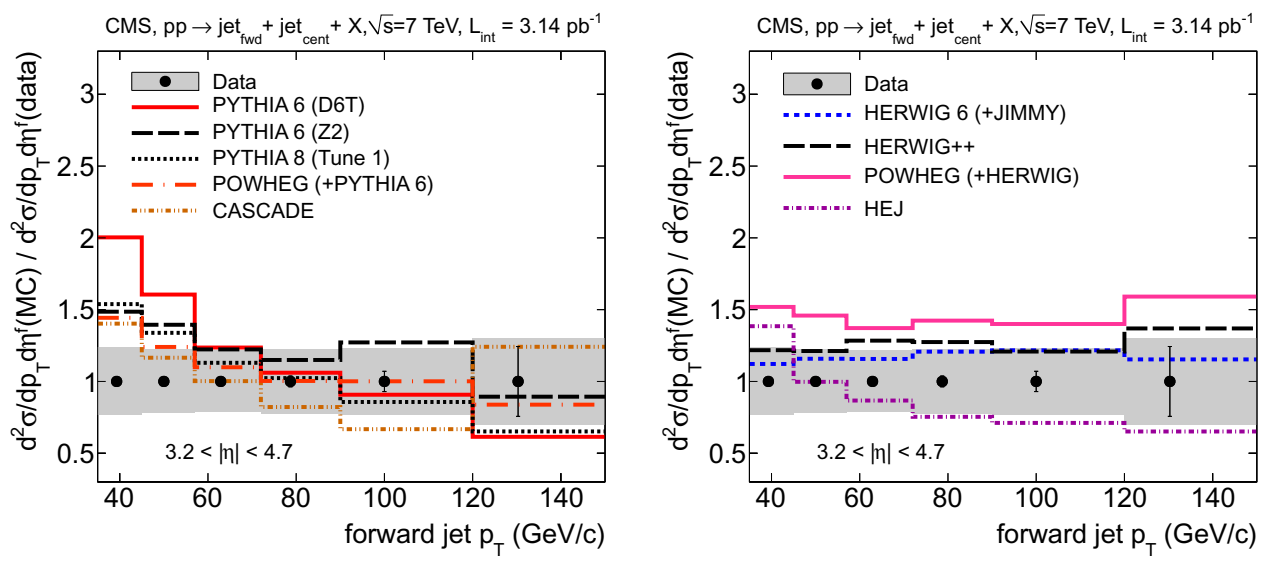

Figure 9. Ratio of theory to data for differential cross sections as a function of $p_{\mathrm{T}}$, for central (left) and forward (right) jets produced in dijet events. The error bars on all data points reflect just statistical uncertainties, with systematic uncertainties plotted as grey bands.

important role in improving the PDFs in the next years to come. Including some of the channels above with the present data typically leads already to $10-15 \%$ improvements of the PDFs in some regions of the Bjorken- $x$ space [39].

Another important uncertainty in e.g. the Higgs cross section predictions at the LHC is the value of the strong coupling constant $\alpha_{\mathrm{s}}$. One of the methods exploited presently at the LHC to determine the strong coupling constant is to use the three-jet to two-jet cross section ratio. CMS presents an analysis [40] where di-jets are used within the range of $420-1390 \mathrm{GeV}$ and the $p_{\mathrm{T}}$ of all jets considered in the study is larger than $150 \mathrm{GeV}$. Measurements of $\alpha_{\mathrm{s}}$ are produced at scales between 400 and $1000 \mathrm{GeV}$. This analysis gives a value of $\alpha_{\mathrm{s}}\left(M_{Z}\right)$ is $0.1148_{-0.0023}^{+0.0055}$ with as most important uncertainties the ones from the PDFs and scale choice.

Among the new analysis techniques that stimulated a lot of interest in the last few years are boosted jets and jet substructure. Indeed, the high energy of the LHC leads to a situation where particles such as the heavy bosons and the top quarks are not produced at rest, but boosted with a high $p_{\mathrm{T}}$ or energy. Also the search for very heavy new particles, with masses in the multi-TeV range, lead to very boosted decay products, even if these are top quarks. This was recognized early on and special workshops [41] have been organized to study boosted techniques in detail. Typically one starts from Cambridge-Aachen fat jets and apply so called jet grooming techniques to find sub-jets. So far many methods have been developed and studied for the analysis of jet substructure and different grooming techniques such as mass drop filtering, trimming, pruning are being pursued. An example of measurements of the $k T$ scale splitting variable $\sqrt{d_{12}}$ is shown in figure 7 (right) [42]. A pioneering paper [43] suggested to use such techniques to resurrect the study of the Higgs to $b b$ decays mode, by un-merging the $b b$ jets from boosted Higgs particles. This technique may become more important at the higher energy running of the LHC. The boosted techniques are now mostly used in the searches for heavy particles. Detailed QCD jet studies such as substructure grooming will be important to get a deeper insight into pQCD evolution in jets and to perform searches for new physics in a new way (figure 8 (right)).

Finally the LHC also allows for QCD studies in extreme regions such as the low Bjorken- $x$ region. In order to probe the low $x$ region a good forward coverage of the experiment is required. CMS made a study of jet measurement with a large rapidity distance, namely the study of correlations between jets with $p_{\mathrm{T}} \geqslant 35 \mathrm{GeV}$ in barrel and forward regions. As an example the correlation between central and forward jets is shown in figure 9 [44]. No model describes all features of these measurements in a satisfactory way. We are now at the start of getting results in such extreme regions and there may be still a lot of surprises laying ahead of us.

\section{The future}

Future possible hadron and lepton colliders will be excellent QCD explorers, and are discussed elsewhere at this Symposium, be it mostly for their capabilities to measure 
the Higgs particle in detail or for the reach of discovering new hypothetical heavy particles. Here we briefly mention the hybrid electron-proton colliders. HERA was the pioneering project that took data for 15 years at DESY, Hamburg, and its biggest legacy is the major improvement in understanding the proton structure. That knowledge is now of extremely high value at the $\mathrm{LHC}$ where the parton distributions are key for searches and precision measurements.

Possible projects for dedicated new facilities of the HERA-type include the LHeC (Europe) and EIC (US). Presently these projects aim for high energy and/or high luminosity $\left(10^{34}-10^{35} \mathrm{~cm}^{-2} \mathrm{~s}^{-1}\right)$ lepton-hadron colliders.

The $\mathrm{LHeC}$ project [45] proposes to add an electron ring or electron linac to the LHC collider, for collisions with energies $60 \mathrm{GeV}$ electrons on $7000 \mathrm{GeV}$ protons. Such a collider should be able to study parton distributions for Bjorken- $x$ with values as low as $10^{-7}$ in the perturbative regime.

The EIC project [46] is less clearly defined right now. One option is to add an electron ring to the Brookhaven RHIC collider, another is to add a proton ring to the JLAB electron ring. The maximum energy of the EIC would be $30 \mathrm{GeV}$ electrons on $250 \mathrm{GeV}$ protons. Polarized beams and high luminosity are the trump cards for this facility.

\section{Summary}

The LHC has provided a wealth of QCD results in its first three years of operation. Understanding of the soft QCD dynamics at the highest energy in the laboratory is mandatory for the physics and for the impact on many other measurements. Also other fields such as cosmic rays studies, can benefit from a thorough and systematic programme of measurements. There are already a lot of measurements available from the experiments, and still a number of phenomena that are insufficiently understood.

Perturbative QCD generally works well for the bulk of the LHC data. The large statistics and high energy of the LHC will allow to explore more extreme phase space corners. But this programme is definitely not yet at the end. New measurements such as jet substructure, low- $x$ measurements, very high $p_{\mathrm{T}}$ and very forward measurement are under way. More measurements will be designed to address some of the questions or concerns mentioned in this review. Finally of course, the new high energy data in 2015 is eagerly waited for, also for new QCD studies.

\section{Acknowledgments}

I am very grateful to the organizers (in particular to Professor Tord Ekelof) for their invitation and hospitality.

\section{References}

[1] Ajaltouni Z J et al 2009 Workshop series on the implications of HERA for LHC physics arXiv:0903.3861

[2] Sjostrand T, Mrenna S and Skands P 2006 J. High Energy Phys. JHEP05(2006)026
[3] Corcella G et al 2002 arXiv:hep-ph/0210213 Bahr M et al 2008 Eur. Phys. J. C 58639

[4] Anelli G et al (TOTEM Collaboration) 2008 J. Instrum. $3 \mathrm{~S} 08007$

[5] Antchev G et al (TOTEM Collaboration) 2013 Europhys. Lett. 10121003

[6] Antchev G et al (TOTEM Collaboration) 2013 Phys. Rev. Lett. 111012001

[7] Chatrchyan S et al (CMS Collaboration) 2013 Phys. Lett. B 7225

[8] Aad G et al (ATLAS Collaboration) 2011 Nature Commun. 2463

[9] Abelev B et al (ALICE Collaboration) 2012 CERN-PH-EP-2012-138 arXiv:1208.4968

[10] Aaij R et al (LHCb Collaboration) 2011 Phys. Lett. B 703267

[11] Chatrchyan S et al (CMS Collaboration) 2012 Eur. Phys. J. C 722080

[12] Aad G et al (ATLAS Collaboration) 2013 New J. Phys. 15033038

[13] Chatrchyan S et al (CMS Collaboration) 2010 J. High Energy Phys. JHEP09(2010)091

[14] Alderweireldt S and Van Mechelen P 2012 arXiv:1203.2048

[15] De Roeck A et al 2002 Eur. Phys. J. C 25391

[16] Boonekamp M, De Roeck A, Peschanski R and Royon C 2002 Phys. Lett. B 55093

[17] Heinemeyer S et al 2011 Eur. Phys. J. C 711649

[18] Albrow M G et al (FP420 Collaboration) 2009 J. Instrum. $4 \mathrm{~T} 10001$

[19] Adriani O (LHCf Collaboration) 2008 J. Instrum. 3 S08006

[20] Aad G et al (ATLAS Collaboration) 2011 Eur. Phys. J. C 711763

[21] Chatrchyan S et al (CMS Collaboration) 2013 Phys. Rev. D 87112002

[22] Aad G et al (ATLAS Collaboration) 2012 Phys. Rev. D 86014022

[23] Aad G et al (ATLAS Collaboration) 2013 Eur. Phys. J. C $\mathbf{7 3} 2509$

[24] Aad G et al (ATLAS Collaboration) 2011 Phys. Rev. Lett. 106172002

[25] Chatrchyan S et al (CMS Collaboration) 2011 Phys. Rev. Lett. 106122003

[26] Chatrchyan S et al (CMS Collaboration) 2012 J. High Energy Phys. JHEP09(2012)094

[27] Buchmuller O, Dolan M J and McCabe C 2013 arXiv:1308.6799

[28] Aad G et al (ATLAS Collaboration) 2012 Eur. Phys. J. C 721993

[29] Aad G et al (ATLAS Collaboration) 2013 J. High Energy Phys. JHEP07(2013)032

[30] Chatrchyan S et al (CMS Collaboration) 2013 Eur. Phys. J. C 732339

[31] Aad G et al (ATLAS Collaboration) 2013 J. High Energy Phys. JHEP06(2013)084

[32] Alekhin S, Blumlein J and Moch S 2012 Phys. Rev. D 86054009

[33] Gao J et al 2013 arXiv:1302.6246

[34] Martin A D, Stirling W J, Thorne R S and Watt G 2009 Eur. Phys. J. C 63189

[35] Ball R et al (NNPDF Collaboration) 2012 Phys. Lett. B 70766

Ball R et al (NNPDF Collaboration) 2011 Nucl. Phys. B 849296

[36] H1 and ZEUS Collaboration $2010 \mathrm{~J}$. High Energy Phys. JHEP01(2010)109

Cooper-Sarkar A M 2012 J. Phys. G: Nucl. Part. Phys. 39093001

[37] Alekhin S et al 2011 The PDF4LHC working group interim report arXiv: 1101.0536

[38] Campbell J 2012 Plenary Talk at ICHEP (Melbourne)

[39] Rojo J 2013 Plenary Talk at DIS (Marseille) 
[40] Chatrchyan S et al (CMS Collaboration) 2013 arXiv: 1304.7498

[41] Altheimer A et al 2012 J. Phys. G: Nucl. Part. Phys. 39063001

[42] Aad G et al (ATLAS Collaboration) 2012 J. High Energy Phys. JHEP05(2012)128

[43] Butterworth J M, Davison A R, Rubin M and Salam G P 2008 Phys. Rev. Lett. 100242001
[44] Chatrchyan S et al (CMS Collaboration) 2012 J. High Energy Phys. JHEP06(2012)036

[45] Abelleira Fernandez J L et al (LHeC Collaboration) 2012 J. Phys. G: Nucl. Part. Phys. 397

[46] Accardi A et al (EIC Collaboration) 2012 The Next QCD Frontier-Understanding the Glue that Binds us All ed A Deshpande, Z-E Meziani and J Qiu (arXiv:1212.1701) 